\title{
MULTINACIONALES DE MERCADOS EMERGENTES Y ADQUISICIONES INTERNACIONALES: EL CASO DE LAS EMPRESAS CHINAS EN EL SECTOR HOTELERO ESPAÑOL
}

\author{
Diego Quer* \\ Universidad de Alicante \\ https://orcid.org/0000-0002-5814-6411 \\ Xing Peng**
}

\section{RESUMEN}

La expansión internacional de las multinacionales de mercados emergentes está adquiriendo una creciente importancia en los últimos años. El sector hotelero no es ajeno a esta tendencia. El objetivo de este trabajo es analizar si los modelos teóricos tradicionales resultan aplicables al caso de las inversiones efectuadas por empresas turísticas chinas o si, por el contrario, se hace necesario utilizar nuevos planteamientos. A partir de un estudio de casos de tres empresas chinas que han invertido en el sector hotelero español, nuestros resultados indican que dichas empresas, más que explotar sus ventajas competitivas, persiguen obtener nuevas fuentes de ventaja. Asimismo, en lugar de seguir un proceso gradual, optan por modos de establecimiento que les permitan acceder de forma rápida a esos activos estratégicos.

Palabras clave: Multinacionales de mercados emergentes; internacionalización; China; sector hotelero español.

Emerging-market multinationals and international acquisitions: The case of Chinese firms in the Spanish hotel industry

Fecha de recepción: 7 de junio de 2018

Fecha de aceptación: 14 de febrero de 2019

*Departamento de Organización de Empresas. Universidad de Alicante. Campus de San Vicente del Raspeig, s/n. 03080 ALICANTE (España).E-mail: diego.quer@ua.es

**Doctora por la Universidad de Alicante. Programa de Doctorado en Dirección y Planificación del Turismo. Instituto Universitario de Investigaciones Turísticas. E-mail: xingpengspain@gmail.com 


\begin{abstract}
The international expansion of emerging-market multinationals is becoming increasingly important in recent years. This trend is also present in the hotel industry. The aim of this paper is to analyze whether traditional theoretical frameworks are applicable to cross-border investments by Chinese tourism companies or new approaches become necessary. From a multiple case study of three Chinese companies with investments in the Spanish hotel industry, our results indicate that these companies, rather than exploiting their competitive advantages, seek to obtain new sources of advantage. Moreover, instead of following a gradual process, they choose establishment modes that allow them to quickly access these strategic assets.
\end{abstract}

Keywords: Emerging-market multinationals; internationalization; China; Spanish hotel industry.

\title{
1. INTRODUCCIÓN
}

Desde el inicio del presente siglo, la expansión internacional de las empresas multinacionales de mercados emergentes se está erigiendo en un fenómeno de creciente relevancia. Entre esas nuevas multinacionales destacan especialmente las procedentes de China, ya que su actividad internacional ha crecido exponencialmente y de forma muy rápida en la última década. De hecho, los flujos de inversión directa en el exterior (IDE) efectuados por empresas chinas se han multiplicado por seis entre 2007 y 2017, hasta el punto de que China ya es el tercer inversor a nivel mundial, solo por detrás de Estados Unidos y Japón (UNCTAD, 2018).

Motivadas esencialmente por la búsqueda de mercados y/o activos estratégicos, las multinacionales chinas han efectuado inversiones significativas en países desarrollados durante los últimos años. El sector turístico no está siendo ajeno a esta tendencia y emblemáticas marcas occidentales están pasando a ser controladas por empresas chinas. Es el caso de cadenas hoteleras como las francesas Louvre (adquirida por Jin Jiang Hotels) y Accor (participada por la misma Jin Jiang), la norteamericana Carlson-Rezidor, ahora Radisson (adquirida por HNA), la cadena francesa de resorts vacacionales Club Med (adquirida por Fosun), el buscador de viajes online Skyscanner de Reino Unido (adquirido por Ctrip), el tour-operador británico Thomas Cook (participado también por Fosun) o la empresa suiza de handling aeroportuario Swissport (ahora en manos de HNA).

Esa creciente presencia de las multinacionales de mercados emergentes está reconfigurando el escenario global. Estas empresas, en particular, las chinas, exhiben un comportamiento diferente al de sus homónimas de los países desarrollados. Ello se manifiesta, por ejemplo, en una menor aversión al riesgo o en una menor influencia de la distancia cultural a la hora de tomar decisiones de localización o elegir el modo de entrada en otros países (Kolstad y Wiig, 2012; Ramasamy et al., 2012). Detrás de ese comportamiento menos convencional, se encuentran factores como la necesidad de acelerar su proceso de 
internacionalización asumiendo mayores riesgos para ponerse al nivel de sus competidores de países desarrollados (Ge y Ding, 2009; Luo y Tung, 2007), las relaciones diplomáticas entre China y algunos países de destino que pueden mitigar los riesgos percibidos (Child y Marinova, 2014; Duanmu, 2014), o la propia influencia institucional del gobierno chino, que les puede llevar a perseguir objetivos que no se guíen solo por la lógica de maximizar beneficios (Buckley et al., 2007, 2016).

Todo ello está llevando a cuestionar algunos de los planteamientos convencionales sobre la IDE y el comportamiento de las empresas multinacionales que se han venido utilizando desde hace décadas. Dichos marcos teóricos surgieron a partir de la observación de las multinacionales procedentes de países desarrollados durante el pasado siglo. Por tanto, esas asunciones que subyacen en los marcos teóricos tradicionales necesitan ser reevaluadas para investigar cómo los atributos específicos de estas nuevas multinacionales de mercados emergentes pueden influir sobre su expansión internacional (Kim y Aguilera, 2016).

Ante el reto que representa este fenómeno, existen diversas posturas contrapuestas que han generado un debate epistemológico entre los investigadores. Así, mientras algunos argumentan que el análisis de estas multinacionales de economías emergentes requiere desarrollar nuevas teorías (Mathews, 2006), otros argumentan que no se necesitan, puesto que los marcos teóricos convencionales también les resultan aplicables (Rugman, 2010). Una tercera postura intermedia sugiere que estas nuevas multinacionales pueden ayudar a extender las teorías existentes, es decir, que su estudio puede contribuir a identificar algunos de los supuestos tácitos sobre los que se construyeron dichas teorías y así extender su capacidad predictiva (Cuervo-Cazurra, 2012). Por consiguiente, resultan necesarias investigaciones adicionales que permitan aportar nueva evidencia empírica ante este debate. Además, los estudios realizados hasta el momento sobre la aplicabilidad de los modelos tradicionales al caso de las multinacionales chinas se han centrado en otros sectores distintos al turístico (Quer et al., 2015). Por ello, es importante analizar si el hecho de tratarse de un sector de servicios influye o no sobre la utilidad de esos planteamientos convencionales.

Hasta el momento, España no ha sido un destino tradicional para las multinacionales chinas. Sin embargo, desde el año 2010 esta tendencia está cambiando de forma importante y algunas empresas chinas han comenzado a realizar importantes inversiones en España. Se están dirigiendo esencialmente a sectores donde España cuenta con know-how avanzado y experiencia, algo que están buscando conseguir muchas empresas chinas. También se interesan por sectores para los que España constituye un importante mercado potencial u ofrece ventajas estratégicas, dada su localización geográfica y lazos históricos y culturales con otros países. Es el caso de la gestión de servicios públicos, la energía, la alimentación, las telecomunicaciones o los servicios logísticos y portuarios. Aunque ya ha habido algunas operaciones, tal y como analizaremos posteriormente, resulta paradójico que el sector turístico y, en particular, el hotelero, no haya sido todavía uno de los objetivos prioritarios de las empresas chinas en España, pese al potencial de mercado que supone como segundo destino turístico mundial y el gran nivel de recursos y capacidades especializados que acumulan las empresas turísticas españolas, incluso a nivel internacional. Una de las posibles razones para ello podría ser que las empresas turísticas que efectúan IDE lo suelen hacer principalmente en aquellos destinos visitados con mayor frecuencia por turistas de su mismo país de origen. En el caso de España, las cifras de visitantes chinos 
todavía no han alcanzado las de otros destinos europeos, aunque están aumentando en los últimos años. De hecho, durante 2017 visitaron España 513.725 turistas chinos, lo que supuso un incremento del $37 \%$ respecto al año anterior (INE, 2018).

Por otra parte, los estudios realizados hasta el momento sobre las inversiones chinas en España no han abordado de forma específica las dirigidas al sector turístico (Carril y Milgram 2016; Casaburi, 2016; Hernández y Álvarez 2009; Quer et al., 2017; Sáez 2010; Santacana y Wang 2008). Además, los escasos trabajos sobre turismo chino en España tampoco han analizado las inversiones efectuadas por empresas chinas en el sector turístico español (Andreu et al., 2009; Hernández, 2018; Lojo, 2016; Lojo y Li, 2018). Por consiguiente, resulta interesante analizar estas inversiones incipientes, máxime cuando China está reorientando su modelo de desarrollo económico hacia sectores de servicios, para lo que necesita know-how avanzado y conocimientos, lo cual abre oportunidades interesantes para las empresas turísticas españolas.

Por tanto, el objetivo de nuestro trabajo es analizar si los modelos teóricos tradicionales resultan aplicables al caso de las inversiones efectuadas por empresas chinas en el sector turístico español o si, por el contrario, se hace necesario utilizar los nuevos planteamientos que han surgido en los últimos años para explicar el comportamiento de las multinacionales de mercados emergentes. Con ello, pretendemos efectuar diversas contribuciones. Desde el punto de vista teórico, buscamos contribuir a ese debate epistemológico mencionado anteriormente. Desde una vertiente empírica, perseguimos aportar nueva evidencia sobre las IDEs efectuadas por empresas chinas en el sector turístico, como sector de servicios que ha recibido una menor atención en estudios anteriores sobre las multinacionales chinas, especialmente en aquellos centrados en España como destino. Asimismo, como contribución práctica, intentaremos aportar algunas claves sobre las oportunidades de cooperación que se abren en un sector como el turístico, donde confluye el elevado nivel de competitividad internacional de España con la necesidad de China de acceder a know-how especializado.

El resto del trabajo se estructura del siguiente modo. En primer lugar desarrollamos el marco teórico atendiendo a esa doble vertiente: teorías tradicionales y nuevos planteamientos. Posteriormente, describimos la naturaleza de nuestro trabajo empírico centrado en un estudio de casos de empresas chinas que han invertido en el sector hotelero español. Tras discutir los resultados obtenidos, ofrecemos una serie de conclusiones.

\section{MARCO TEÓRICO}

\subsection{Paradigma Ecléctico y Modelo LLL (Linkage, Leverage, Learning)}

Entre las distintas justificaciones tradicionales sobre la existencia de la empresa multinacional destaca el Paradigma Ecléctico de Dunning (1980, 1988, 1995, 2000), también conocido como Modelo OLI, acrónimo de Ownership (propiedad), Location (localización) e Internalization (internalización). Según este planteamiento, una empresa solo efectuará IDE y, por tanto, se convertirá en multinacional, si se confluyen simultáneamente tres ventajas: (1) la empresa posee una ventaja competitiva previa que le permite compensar las desventajas de operar en un mercado extranjero (ventaja en propiedad); (2) existe algún factor vinculado al país de destino que lo hace más atractivo frente a otros destinos poten- 
ciales (ventaja de localización); y (3) resulta más beneficioso para la empresa explotar de manera directa esas ventajas antes que hacerlo a través de un acuerdo contractual con una empresa externa, evitando así costes de transacción (ventaja de internalización).

Cabe destacar que el Paradigma Ecléctico también ha sido aplicado al caso del sector hotelero por Dunning y McQueen (1982a, 1982b), quienes efectuaron algunas matizaciones para adaptarlo a ese contexto específico. Respecto a las ventajas en propiedad, enfatizan aquellas que pueden reportar una ventaja competitiva específica en el sector, tales como: conocimiento del mercado turístico gracias a la experiencia acumulada en el país de origen o en otros destinos; imagen de marca; habilidad para innovar y ofrecer servicios complementarios; tamaño y grado de diversificación de la empresa; etc. En cuanto a las ventajas de localización, señalan algunos factores que pueden hacer atractivo un país para la implantación hotelera: tamaño y tasa de crecimiento del sector; infraestructura existente para el turismo; disponibilidad y calidad de los inputs hoteleros; política gubernamental hacia la inversión extranjera; estabilidad política, social y económica; actitud de la población local hacia los turistas extranjeros; etc. Es en el tercer ingrediente, las ventajas de internalización, donde reinterpretan en cierta medida el modelo, argumentando que, además de la posesión de un porcentaje de propiedad suficiente, los acuerdos contractuales (alquiler, contratos de gestión o franquicias) también permiten a una empresa hotelera dirigir y controlar sus operaciones en el extranjero, evitando así algunos costes de transacción.

En cualquier caso, el Paradigma Ecléctico es una de las teorías tradicionales que se formularon inicialmente para explicar las IDEs dirigidas a explotar activos por parte de empresas de países desarrollados que poseían ventajas en propiedad significativas, no para aquellas IDEs que persiguen explorar nuevos activos, como están haciendo muchas multinacionales de mercados emergentes en años recientes (Strange, 2018). En otras palabras, esos marcos teóricos tradicionales están estrechamente ligados a la idea de la existencia de una ventaja competitiva previa como requisito necesario para la internacionalización (Child y Rodrigues, 2005).

Por consiguiente, el Paradigma Ecléctico es uno de esos planteamientos convencionales que está siendo objeto de debate, tal y como comentamos anteriormente. Algunos investigadores como Narula (2012) argumentan que hay un principio fundamental que resulta aplicable a todas las multinacionales, independientemente de su nacionalidad: existe un umbral mínimo de activos específicos que la empresa debe poseer para una expansión internacional exitosa. Además, según este punto de vista, más allá de las diferentes condiciones iniciales de los países de origen, conforme esas multinacionales de mercados emergentes vayan evolucionando, las diferencias respecto a las multinacionales de países avanzados tenderán a disminuir. Sin embargo, otros autores adoptan una visión mucho más rupturista. Así, aun admitiendo que las multinacionales de mercados emergentes poseen algunos activos específicos que pueden explotar en mercados extranjeros, Hennart (2012) argumenta que el Paradigma Ecléctico no permite explicar adecuadamente las IDEs efectuadas por dichas multinacionales, en particular, aquellas dirigidas a la búsqueda de activos intangibles en países desarrollados.

Es en este contexto donde han surgido recientemente algunos nuevos planteamientos para explicar de forma específica ese proceso de internacionalización de las empresas de mercados emergentes, y que pueden constituir un reto para los marcos teóricos tradiciona- 
les. Uno de ellos es el Modelo LLL (acrónimo de Linkage-Leverage-Learning) propuesto por Mathews (2006). Su idea central es que esas multinacionales procedentes de países emergentes son empresas recién llegadas a la escena global y, por tanto, no dependen para su expansión internacional de la posesión previa de recursos, como fue el caso de muchas multinacionales de países desarrollados años atrás. Por el contrario, estas nuevas multinacionales utilizan la expansión internacional para acceder a recursos que de otro modo no estarían disponibles. A partir de ello, el Modelo LLL se articula en torno a tres pilares fundamentales: (1) Linkage o vínculo (el punto de partida fundamental para estas empresas es que no se están centrando en sus propias ventajas, sino en los recursos a los que van a ser capaces de acceder desde el exterior, por lo que una orientación global buscando establecer vínculos con otras empresas se convierte en una fuente de ventajas); (2) Leverage o aprovechamiento (formas en que se pueden utilizar o explotar los recursos, algo relacionado con el grado en que dichos recursos son accesibles); (3) Learning o aprendizaje (la aplicación repetida de los dos procesos anteriores puede resultar en que la empresa aprenda a realizar estas operaciones de manera más eficiente, es decir, que consiga aprendizaje organizacional).

En definitiva, la idea esencial del Modelo LLL es que las multinacionales de economías emergentes se encuentran rezagadas frente a las multinacionales ya establecidas, por lo que la aplicación repetida de los procesos de vínculo, aprovechamiento y aprendizaje puede permitirles reducir dicha desventaja. Este proceso de construcción puede ayudar a explicar por qué estas empresas han aparecido en los mercados internacionales de forma tan repentina y han seguido un proceso de internacionalización tan acelerado, tal y como se ha constatado en algunas investigaciones previas sobre las multinacionales chinas (Ge y Ding, 2008).

Ante esta propuesta de reconsiderar el Paradigma Ecléctico, el propio Dunning (2006) respondió aceptando que los determinantes de la IDE que busca aumentar activos encajan mejor con la idea de que, al menos, algunas de las ventajas competitivas de las empresas "siguen" a la internacionalización más que "conducen" a ella. No obstante, argumentó que, para llevar a cabo ese tipo de IDE, la empresa tiene que poseer ciertas ventajas únicas (que pueden ser específicas de la empresa o de su país de origen). Por ejemplo, en el caso de las multinacionales chinas, podrían ser la mayor facilidad para obtener la financiación necesaria para adquirir una empresa extranjera. En cualquier caso, cada uno de los modelos presenta sus respectivos sesgos: hacia el interior de la empresa (el Paradigma Ecléctico) y hacia el exterior (el marco LLL). Por ello, y con la finalidad de obtener una visión más equilibrada, $\mathrm{Li}$ (2007) propone una integración de ambos modelos para una mejor explicación de todos los tipos de IDE efectuados tanto por multinacionales procedentes de países desarrollados como de economías emergentes.

\subsection{Modelo del Proceso y Perspectiva Trampolín}

Otro de los planteamientos tradicionales más influyentes es el Modelo del Proceso, también conocido como Modelo de Uppsala (Johanson y Wiedersheim-Paul, 1975; Johanson y Vahlne, 1977, 1990). Este enfoque considera que muchas empresas inician sus operaciones internacionales cuando son comparativamente pequeñas, desarrollando gradualmente sus operaciones exteriores. Por tanto, frente al estudio de las operaciones de empresas que ya 
son grandes e internacionales, su énfasis recae en la explicación del desarrollo completo que les ha conducido a obtener un determinado nivel de internacionalización. Ese proceso gradual, observado originalmente en una serie de empresas suecas, parte de dos asunciones básicas: (1) la empresa se desarrolla primero en su mercado doméstico; (2) los obstáculos más importantes para la internacionalización son la falta de conocimientos y de recursos, que son reducidos a través de una toma de decisiones y un aprendizaje incrementales.

Debido a esos obstáculos, el inicio de la expansión internacional está condicionado por dos elementos importantes. Por un lado, la distancia psíquica, referida a factores como las diferencias en lengua, cultura, sistemas políticos, nivel de educación, nivel de desarrollo, etc. Según el Modelo del Proceso, las empresas entrarán primero en países que sean comparativamente similares, al tratarse, en principio, de entornos más familiares. Por otro lado, el tamaño del mercado potencial; en este caso, la empresa preferirá empezar sus operaciones en mercados pequeños donde se precise un menor compromiso inicial de recursos o donde exista una menor competencia. Partiendo de estas dos ideas, el proceso de internacionalización puede transcurrir por una senda denominada "cadena de establecimiento", constituida por diversas etapas que implican sucesivamente un mayor compromiso de recursos hasta alcanzar la IDE.

Años después de su propuesta original, Johanson y Vahlne (2009) revisaron el Modelo del Proceso para incorporar la importancia de las redes de relaciones entre empresas. Su punto de partida es que el entorno actual para los negocios está configurado por una red de relaciones estrechas y duraderas entre socios. Así, en su modelo revisado introducen el concepto de liability of outsidership (desventaja de estar fuera de la red de relaciones), que se añade a la liability of foreignness (desventaja del extranjero) ya incluida en el modelo original. Con ello, manteniendo la idea de un proceso gradual fundamentado en un aprendizaje incremental, este Modelo del Proceso revisado añade varias ideas clave. Por un lado, la pertenencia a una red de relaciones así como la confianza y el compromiso recíprocos entre socios son necesarios para el éxito de la internacionalización. Por otro, el efecto de la "desventaja del extranjero" sigue estando presente en el modelo revisado ya que, a mayor distancia psíquica, más difícil será construir relaciones. Además, si una empresa intenta entrar en un mercado extranjero donde no cuenta con una posición relevante en ninguna red, sufrirá tanto la "desventaja de estar fuera de la red de relaciones" como la "desventaja del extranjero".

Al igual que ocurría con el Paradigma Ecléctico, la entrada en el escenario global de las multinacionales de mercados emergentes también está suponiendo un reto para algunas de las ideas subyacentes en el Modelo del Proceso. Así, Liu et al. (2008) defienden que el proceso de internacionalización de las empresas privadas chinas solo puede ser explicado parcialmente por el Modelo del Proceso dado que, en realidad, comienzan su proceso de internacionalización dentro de su propio país de origen. Ello es debido a que muchas de esas empresas efectúan primero operaciones internacionales con empresas extranjeras en China para adquirir los conocimientos tecnológicos y de gestión que necesitan para iniciar sus operaciones internacionales en otros países.

Por otra parte, las multinacionales de mercados emergentes, como recién llegadas a la arena internacional, tienden a acelerar su proceso de internacionalización con el fin de acceder a recursos y capacidades que no están disponibles en su país de origen y que necesitan 
para ponerse al mismo nivel que sus competidores establecidos (Mathews, 2002). Es en este contexto donde ha surgido otro nuevo planteamiento denominado Perspectiva Trampolín (Springboard Perspective), propuesta por Luo y Tung (2007) para explicar el comportamiento de estas multinacionales, en particular, cuando se dirigen a países desarrollados. Su idea central es que dichas empresas consideran a menudo la expansión internacional como un trampolín para obtener los activos estratégicos que necesitan con la finalidad de dar alcance a sus competidores de países más avanzados. Ello les lleva en ocasiones a entrar de forma anticipada en países con los que existe una elevada distancia psíquica, contrariamente a lo que postulan planteamientos convencionales como el Modelo del Proceso.

Además, frente a la creación de nuevas filiales propias (inversiones greenfield), suelen optar por adquisiciones de empresas establecidas en países desarrollados, ya que dicho modo de establecimiento favorece un acceso más rápido a esos activos estratégicos que necesitan (Child y Rodrigues, 2005; Globerman y Shapiro, 2009). En definitiva, estas empresas tratan de superar sus desventajas como recién llegadas mediante adquisiciones, las cuales pueden suponer un mayor riesgo potencial (Kedia et al., 2012). Además de implicar habitualmente la necesidad de efectuar la inversión de una sola vez, frente a la posibilidad de escalonarla en caso de nueva filial propia, entran en escena las dificultades post-adquisición derivadas de la integración de la empresa adquirida, especialmente en un país con el que suele existir una elevada distancia psíquica (Dikova y Van Witteloostuijn, 2007). Todo ello hace que esta Perspectiva Trampolín sugiera que las multinacionales de economías emergentes pueden saltar rápidamente desde su posición inicial de recién llegadas hasta una posición competitiva más avanzada. Por consiguiente, tienden a internacionalizarse rápidamente y no de modo incremental como sostiene el Modelo del Proceso (Luo y Tung, 2007).

Una década después de su propuesta inicial, Luo y Tung (2018) actualizaron esta Perspectiva Trampolín, introduciendo lo que ellos denominan modelo de espiral ascendente. Así, sostienen que estas nuevas multinacionales comienzan con una internacionalización interior, es decir, dentro de su propio país de origen, tal y como comentamos anteriormente. Después, efectúan IDEs de modo arriesgado, transfieren los activos adquiridos a su país de origen para mejorar su dotación de recursos y, sucesivamente, se catapultan globalmente con mayores capacidades.

\section{METODOLOGÍA}

Nuestro estudio empírico se ha centrado en las inversiones realizadas por las empresas chinas en el sector hotelero español. Dicho contexto resulta apropiado dados los objetivos de nuestra investigación, al tratarse de IDEs realizadas por empresas procedentes de una economía emergente en un país desarrollado que cuenta con recursos y capacidades avanzados en el sector. Además, dado que, hasta el momento, es todavía escaso el número de empresas chinas que han invertido en el sector hotelero español, hemos optado por el estudio de casos como metodología de investigación cualitativa (Yin, 1981).

Como hemos indicado en el apartado anterior, no existe consenso acerca de la validez de las teorías tradicionales o la necesidad de aplicar los nuevos marcos conceptuales que han surgido a la hora de explicar el comportamiento de las multinacionales de mercados emergentes. Por ello, hemos realizado un estudio de casos de tipo exploratorio, que 
trata de examinar un fenómeno para el que no existe un marco teórico bien definido, con la finalidad de poder desarrollar proposiciones teóricas que permitan, en última instancia, seguir generando teoría sobre un determinado tema (Eisenhardt, 1989; Yin, 2014). Además, se trata de un estudio de casos comparativo o múltiple, en los que se suelen hacer las mismas preguntas para así comparar las respuestas obtenidas y poder extraer conclusiones.

Se han analizado los casos de las tres únicas empresas chinas que, hasta la fecha de realización del estudio empírico en el año 2016, habían efectuado alguna inversión en el sector hotelero español: HNA Hospitality Group (HNA), Jiangsu GPRO Group Co., Ltd. (GPRO) y Chongqing Kangde Industrial Group Co., Ltd. (Kangde). Es preciso destacar que nos hemos centrado solo en empresas de China continental, ya que existe una cadena de Hong Kong (Mandarin Oriental Hotel Group) que también cuenta con dos hoteles en España. La tabla 1 presenta una breve descripción las tres empresas objeto de estudio.

Tabla 1

DESCRIPCIÓN DE LOS CASOS DE ESTUDIO

\begin{tabular}{|c|c|c|c|c|c|}
\hline $\begin{array}{c}\text { Empresa } \\
\text { china }\end{array}$ & $\begin{array}{c}\text { Sede central } \\
\text { corporativa }\end{array}$ & $\begin{array}{c}\text { Tipo de } \\
\text { propiedad }\end{array}$ & Empleados & $\begin{array}{c}\text { Año de } \\
\text { fundación }\end{array}$ & $\begin{array}{c}\text { Sectores } \\
\text { principales }\end{array}$ \\
\hline HNA & Hainan & No estatal & 120.000 & 1997 & $\begin{array}{c}\text { Hoteles, } \\
\text { líneas aéreas, } \\
\text { intermediación }\end{array}$ \\
\hline GPRO & Jiangsu & No estatal & 10.000 & 2004 & $\begin{array}{c}\text { Inmobiliario, } \\
\text { servicios }\end{array}$ \\
\hline Kangde & Chongqing & No estatal & 1.200 & 1997 & $\begin{array}{c}\text { Inmobiliario, } \\
\text { hoteles, deporte }\end{array}$ \\
\hline
\end{tabular}

Se ha obtenido información primaria fundamentalmente a partir de entrevistas a altos directivos de las empresas seleccionadas, algunas de ellas realizadas de forma personal en China por uno de los autores del trabajo entre los meses de marzo y mayo de 2016. Se aplicó un procedimiento de entrevista semi-estructurada para facilitar la obtención de información (Walsh et al., 1999). Utilizamos esencialmente preguntas abiertas que dan a los informantes margen para exponer con mayor amplitud las cuestiones abordadas. Además, para obtener una información más completa solicitamos a los entrevistados que proporcionaran más detalles cuando sus descripciones eran demasiado breves o cuando surgían nuevos aspectos a desarrollar (Eisenhardt y Graebner, 2007). En cada entrevista se siguió un guion en torno a dos grandes boques: (1) razones para elegir España como destino dentro de Europa; (2) motivo concreto para invertir en España y ventajas competitivas percibidas (relacionándolo con las teorías tradicionales y los nuevos marcos teóricos para las multinacionales de economías emergentes).

Además de estas entrevistas personales, la información se completó mediante contactos con las personas entrevistadas y otros directivos de las empresas analizadas a través de teléfono, correo electrónico y WeChat (la red social más utilizada en China). Asimismo, se utilizó información secundaria procedente de la web corporativa de cada empresa así 
como noticias publicadas en diversos medios. Este procedimiento de triangulación permite obtener información más precisa y mejorar la validez de los resultados obtenidos con un estudio de casos (Anand et al., 2007).

\section{RESULTADOS Y DISCUSIÓN}

Como primera aproximación a los casos objeto de estudio, vamos a ofrecer una breve descripción de las inversiones realizadas en España por las tres empresas chinas. En primer lugar, destaca que, en los tres casos, han efectuado adquisiciones de empresas ya establecidas, aunque encontramos algunas diferencias. Así, mientras HNA adquirió un porcentaje significativo del capital de una de las cadenas hoteleras españolas más importantes $(\mathrm{NH})$, las otras dos empresas chinas (GPRO y Kangde) compraron sendos establecimientos hoteleros individuales, localizados en Mallorca y Tenerife, respectivamente. No obstante, cabe destacar que, en estos dos casos, las formas de administración y gestión son muy diferentes. Mientras GPRO sí iba a gestionar el establecimiento adquirido (Hotel Valparaíso Palace \& Spa de Mallorca), Kangde adquirió el Hotel Barceló Santiago de Tenerife, pero la cadena española Barceló iba a continuar gestionando el hotel. Por otra parte, HNA ya contaba con cierta presencia en otros países mientras que, para GPRO y Kangde, la entrada en España supuso su primera incursión internacional. La tabla 2 resume los principales datos descriptivos de las tres inversiones.

Tabla 2

CARACTERÍSTICAS DE LAS INVERSIONES CHINAS ANALIZADAS

\begin{tabular}{|c|c|c|c|c|}
\hline $\begin{array}{c}\text { Empresa } \\
\text { china }\end{array}$ & $\begin{array}{c}\text { Año de la } \\
\text { inversión }\end{array}$ & $\begin{array}{c}\text { Modo de } \\
\text { establecimiento }\end{array}$ & Empresa objetivo & $\begin{array}{c}\text { Presencia } \\
\text { internacional } \\
\text { previa }\end{array}$ \\
\hline HNA & $2013-2014$ & $\begin{array}{c}\text { Adquisición parcial } \\
(29,5 \%)\end{array}$ & NH Hotel Group & Sí \\
\hline GPRO & 2014 & Adquisición total & $\begin{array}{c}\text { Hotel Valparaíso } \\
\text { Palace \& Spa } \\
\text { (Mallorca) }\end{array}$ & $\begin{array}{c}\text { No (primera IDE } \\
\text { en España) }\end{array}$ \\
\hline Kangde & 2014 & Adquisición total & $\begin{array}{c}\text { Hotel Barceló } \\
\text { Santiago (Tenerife) }\end{array}$ & $\begin{array}{c}\text { No (primera IDE } \\
\text { en España) }\end{array}$ \\
\hline
\end{tabular}

Como ya se ha comentado, la primera parte de cada entrevista giró en torno a las razones que habían llevado a cada empresa a decantarse por España como destino dentro de Europa. En los tres casos hubo coincidencia en señalar como motivo fundamental las ventajas de España como destino turístico consolidado dentro de Europa, enfatizando atractivos como el clima, el patrimonio cultural, la hospitalidad o la buena localización. Así lo puso de manifiesto de forma explícita el Vicepresidente Ejecutivo de GPRO, al señalar que "la gente es abierta, el clima es estupendo, hay casi 300 días de sol al año" y que "España posee una rica experiencia vacacional cultural. Es el complejo turístico 
vacacional de Europa". De forma similar, el Director del Departamento de Inversiones en el Exterior de Kangde, apuntó que el Hotel Barceló Santiago de Tenerife que habían adquirido "está situado estratégicamente en una de las principales islas turísticas de España y de Europa".

En cualquier caso, también hemos identificado algunas razones específicas por parte de cada empresa. Así, el Director General de Recursos Humanos de HNA hizo hincapié en la necesidad de fortalecer la presencia internacional del grupo en una región donde, hasta ese momento, no tenían presencia. Por su parte, desde GPRO apuntaron a la pretensión de aprovechar el crecimiento del número de turistas chinos en España, ofreciéndoles un hotel con servicios adaptados a sus necesidades. Igualmente, en GPRO se hizo referencia a la influencia institucional del gobierno chino en su decisión de desembarcar en España a través de la denominada Belt \& Road initiative o iniciativa de la Franja y la Ruta, también rebautizada como la Nueva Ruta de la Seda. Este es un ambicioso proyecto del gobierno chino iniciado en 2013 que pretende fomentar la cooperación internacional en varias áreas: comercio e inversión, infraestructuras, finanzas, políticas e intercambio cultural. Si bien el turismo no es uno de los sectores integrado específicamente en la Franja y la Ruta, llama la atención la influencia que está teniendo esta iniciativa como catalizadora de los procesos de expansión internacional de las empresas chinas de muchos sectores. De hecho, el alto directivo de GPRO entrevistado apuntó que "su inversión en el sector hotelero español nace de una reflexión y una respuesta al llamamiento del Estado en lo que respecta a la iniciativa de la Franja y la Ruta".

Como ya hemos indicado, el segundo bloque de las entrevistas giró en torno a los motivos concretos de cada una de las inversiones, a través de una serie de preguntas relacionadas con los marcos teóricos tradicionales (Paradigma Ecléctico y Modelo del Proceso) y los nuevos planteamientos alternativos (Modelo LLL y Perspectiva Trampolín).

En relación con el Paradigma Ecléctico, tal y como ya se desprendía del primer bloque de preguntas, las tres empresas chinas han enfatizado las ventajas de localización que supone España como destino turístico consolidado dentro de Europa. Respecto a las ventajas en propiedad, de sus respuestas no se desprende que la intención de alguna de esas tres empresas haya sido explotar en España alguna ventaja competitiva que ya poseían. Como ya hemos señalado, HNA sí contaba con experiencia previa en el sector tanto en China como en otros países, pero las otras dos empresas no contaban con presencia internacional anteriormente. Además, aunque Kangde sí tenía cierta experiencia en el negocio hotelero dentro de China, para GPRO, la adquisición del Hotel Valparaíso Palace \& Spa de Mallorca supuso, no solo su primera operación internacional, sino también su primera incursión en el negocio hotelero. La actividad de GPRO se ha centrado tradicionalmente en negocios inmobiliarios y de servicios y, aunque en China ya tenía una empresa de gestión hotelera, todavía no contaban con ningún hotel en el momento de su entrada en España. Las ventajas de internalización tampoco parecen ser determinantes, ya que solo el alto directivo de HNA sugirió que su decisión de invertir directamente les permitía aprovechar las ventajas de un control directo de sus operaciones en España.

Por contra, los directivos entrevistados sí parecen coincidir en que sus decisiones de inversión en España han estado motivadas por la pretensión de acceder a algún recurso o capacidad que no poseían previamente, tal y como propone el Modelo LLL. En el caso 
de HNA, su Director General de Recursos Humanos señaló que su empresa objetivo (NH) "tiene una sólida y amplia experiencia operativa así como un sistema de gestión internacional que ofrece una buena imagen de marca". Además, sus hoteles de negocios de gama media-alta encajan perfectamente en la estrategia de desarrollo que pretende impulsar HNA". Por tanto, el establecimiento de vínculos con NH para acceder a esos recursos fue el punto de partida de la decisión de HNA de invertir en España. Se trataba, por tanto, de unos recursos de los que carecía HNA en su mercado doméstico y que trataba de buscar en el exterior. Además, dichos recursos con los que contaba NH eran exactamente una necesidad para HNA como recién llegada al escenario internacional. En cuanto al aprovechamiento, segundo componente del Modelo LLL que se relaciona con el grado de accesibilidad de los recursos para poder explotarlos y aprovecharlos, desde HNA apuntaron a la necesidad de superar algunas barreras para acceder a los conocimientos de la empresa adquirida. En este sentido, el directivo entrevistado señaló que las principales barreras que encontraron fueron "las barreras culturales e idiomáticas" y "recursos humanos con escasa preparación vinculada a la idiosincrasia china". Por lo que respecta al aprendizaje (tercer elemento del Modelo LLL), el directivo de HNA reconocía que, en los próximos años, el grupo debía ir aprendiendo la forma más eficaz en que la experiencia, el sistema de gestión y el conocimiento específico podrían ser difundidos más rápidamente desde los hoteles de NH hacia HNA durante su expansión internacional hacia mercados concretos.

De forma análoga, el Modelo LLL también encaja en el caso de la adquisición del Hotel Valparaíso Palace \& Spa de Mallorca por parte de GPRO. De hecho, su Vicepresidente Ejecutivo enfatizó que "los motivos para invertir en el sector hotelero español no son solamente para llegar a obtener un rendimiento deseado a nivel económico, significa también conseguir la experiencia del negocio hotelero y de la gestión turística vacacional de primera mano". En otras palabras, buscaba establecer vínculos con un hotel consolidado y con experiencia en el segmento alto del negocio hotelero vacacional, que eran exactamente factores clave que encajaban en la necesidad de GPRO como empresa multisectorial que carecía de conocimientos específicos sobre la industria hotelera. Respecto al aprovechamiento, el directivo de GPRO, en línea similar al caso de HNA, señaló algunas barreras que habían encontrado a la hora de acceder a esos recursos, concretamente: "el ambiente de mercado y el pensamiento diferente al nuestro" así como "la legislación (incluyendo el sistema financiero) y los orígenes y el bagaje cultural de España, tan distinto a China". En cuanto al tercer ingrediente del Modelo LLL (aprendizaje), enfatizó la necesidad de "aprender los conceptos y experiencias sectoriales avanzados a través del proyecto de inversión, para mejorar la eficacia de la empresa (económica, sostenible, de recursos humanos, etc.)". En este sentido, resulta muy destacable que, como ya hemos señalado, para GPRO se trataba de su primera incursión efectiva en el negocio hotelero, por lo que los recursos obtenidos a través del vínculo con el Hotel Valparaíso Palace \& Spa de Mallorca podían serle útiles no sólo para su expansión hotelera a nivel internacional, sino también dentro de la propia China.

El caso de la adquisición del Hotel Barceló Santiago de Tenerife por parte de Kangde también encaja en los planteamientos subyacentes en el Modelo LLL. Se trataba del primer paso de su expansión internacional, adquiriendo un hotel que, no solo estaba situado en uno de los principales destinos turísticos españoles sino que, además, permitía establecer 
vínculos con Barceló, otro de los grupos hoteleros españoles más emblemáticos, que seguiría gestionando el establecimiento tras la adquisición. Aunque el Director del Departamento de Inversiones en el Exterior de Kangde no destacó ninguna barrera en particular, como sí hicieron los otros entrevistados, durante el proceso de obtención de datos sí detectamos que para esta empresa china existía la necesidad de establecer mecanismos para un adecuado aprovechamiento tanto de los recursos específicos del hotel de Barceló como de aquellos más genéricos vinculados a estar presente en un destino turístico de referencia como Tenerife. Finalmente, en cuanto al aprendizaje, destaca de nuevo que la búsqueda y asimilación de conocimientos parece ser un factor fundamental en la decisión de inversión de Kangde en España. Tal y como señaló el directivo entrevistado, su intención era fundar una central en Europa y seguir manteniendo negociaciones para posicionarse en otros destinos tanto españoles como europeos con la finalidad de continuar su expansión internacional dentro del sector hotelero.

En definitiva, a partir de los resultados obtenidos del análisis de los tres casos, podemos establecer la siguiente proposición relativa a la disyuntiva entre el Paradigma Ecléctico o el Modelo LLL para explicar las inversiones chinas en el sector hotelero español:

Proposición 1. Las inversiones realizadas por empresas chinas en el sector hotelero español están motivadas esencialmente por los procesos de vínculo, aprovechamiento y aprendizaje que sugiere el Modelo LLL y, en menor medida, por las ventajas propuestas por el Paradigma Ecléctico, ya que solo las ventajas de localización parecen resultar determinantes.

Por lo que respecta al seguimiento de un proceso gradual, tal y como propone el Modelo del Proceso, o una internacionalización más acelerada, como sugiere la Perspectiva Trampolín, aunque existen algunas diferencias derivadas del perfil y la trayectoria anterior de las tres empresas chinas inversoras, los resultados obtenidos sí parecen bastante concluyentes. Por un lado, aunque durante las entrevistas los directivos se mostraban prudentes al referirse a la expansión internacional futura de sus empresas, lo cierto es que las tres han optado por la IDE como primera estrategia de entrada en España, en lugar de por fórmulas contractuales que impliquen un menor compromiso inicial de recursos, al contrario de lo que sugiere el Modelo del Proceso. Además, como ya hemos indicado, para GPRO y Kangde, la entrada en España supuso su primera incursión internacional y, en el caso de HNA, se trataba de su primera decisión de entrada en España.

Por otra parte, los tres casos han optado por la adquisición como modo de establecimiento. Tal y como argumentamos en el apartado teórico, esta es una fórmula más arriesgada que establecer una nueva filial propia, máxime cuando la distancia cultural es relativamente elevada, como ocurre entre China y España. No obstante, de acuerdo a lo ya discutido, las tres empresas chinas buscan activos estratégicos vinculados al sector turístico en España (conocimientos, experiencia, imagen de marca...) y precisamente, la adquisición de una empresa ya establecida resulta un medio más rápido para conseguirlos. Por consiguiente, poca aversión al riesgo y un proceso de internacionalización acelerado en lugar de por etapas son características que encajan mejor en la Perspectiva Trampolín.

Además, de acuerdo a lo que se desprende de las entrevistas y de la información adicional analizada, parece que estas empresas van a utilizar sus inversiones en España como un "trampolín" para profundizar en su proceso de internacionalización (en el caso 
de HNA), para abordar una expansión posterior de mayor calado en Europa (en el caso de Kangde) o incluso para mejorar su posicionamiento en el propio país de origen gracias a los conocimientos avanzados sobre el negocio hotelero (en el caso de GPRO).

Todo ello nos lleva a establecer la siguiente proposición en cuanto a la aplicabilidad del tradicional Modelo del Proceso o de la nueva Perspectiva Trampolín para multinacionales de mercados emergentes:

Proposición 2. En sus decisiones de inversión en el sector hotelero español, las empresas chinas no han seguido una aproximación gradual tal y como sugiere el Modelo del Proceso, sino que, de acuerdo a la Perspectiva Trampolín, han optado por realizar directamente adquisiciones, lo que les permite un acceso más rápido a los activos estratégicos que necesitan para ponerse al nivel de sus competidores de países desarrollados.

\section{CONCLUSIONES}

Nuestro estudio ha pretendido analizar si los modelos teóricos tradicionales, que surgieron a raíz de la expansión internacional de multinacionales de países desarrollados, resultan aplicables a al caso de las inversiones chinas en el sector hotelero español o si, por el contrario, resulta necesario utilizar los nuevos planteamientos que han surgido para explicar el comportamiento de las multinacionales de mercados emergentes. Los resultados de nuestro estudio de casos sugieren lo segundo ya que, dichas empresas, con sus inversiones en España, más que explotar sus ventajas competitivas existentes (como sostiene el tradicional Paradigma Ecléctico), persiguen acceder a nuevas fuentes de ventaja (como sugiere el nuevo Modelo LLL). Además, en lugar de seguir un proceso gradual (como defiende el tradicional Modelo del Proceso), optan directamente por modos de establecimiento más arriesgados pero que les permitan acceder de forma rápida a esos activos estratégicos que precisan para mejorar su competitividad internacional (como propone la nueva Perspectiva Trampolín).

Consideramos que nuestro trabajo ha efectuado las siguientes contribuciones. Desde un punto de vista teórico, hemos contribuido a ese debate epistemológico todavía no resuelto sobre la disyuntiva entre teorías tradicionales y nuevos planteamientos que, en años recientes, está adquiriendo gran relevancia en la literatura sobre negocios internacionales ante la creciente expansión internacional de las empresas de mercados emergentes. Desde el punto de vista empírico, hemos aportado nueva evidencia para esa disyuntiva en el caso de las inversiones chinas en el sector hotelero (sector menos abordado en estudios anteriores sobre multinacionales chinas) y además centrándonos en España (donde los trabajos realizados hasta ahora sobre turismo chino no han analizado de forma específica las inversiones chinas en el sector).

Asimismo, consideramos que nuestro estudio tiene también algunas implicaciones prácticas, tanto a nivel empresarial como institucional. Tal y como se ha puesto de manifiesto en nuestro estudio de casos, el sector turístico es precisamente uno de los campos donde más se puede profundizar en la cooperación entre España y China de cara al futuro. En los últimos años, China está intentando reorientar su modelo económico, desde la tradicional exportación de manufacturas de bajo coste hacia un nuevo modelo donde los sectores de servicios y el conocimiento adquieran cada vez más importancia. Es en este 
contexto donde España tiene mucho que ofrecer en materia turística, dado el gran nivel de experiencia, conocimientos avanzados e imagen de marca que acumulan sus empresas. La búsqueda de esos activos estratégicos en España ha sido precisamente el eje central de los tres casos de empresas chinas que hemos analizado. Es preciso destacar que otras empresas hoteleras chinas han buscado acceder a ese know-how español en su propio país. Es el caso de la cadena Jin Jiang Hotels, que estableció una alianza estratégica con Meliá Hotels International, en virtud de la cual la cadena española ya gestiona varios hoteles en China.

Como contraprestación a ese trasvase de recursos intangibles, la presencia de empresas hoteleras chinas en España puede atraer a un mayor número de turistas chinos. Adicionalmente, la entrada de capital chino en esas empresas españolas podría tener el efecto inducido de propiciar oportunidades de negocio en China, donde las cadenas hoteleras españolas podrían también explotar su know-how en un destino turístico cada vez más importante. Como botón de muestra, cabe mencionar uno de nuestros casos de estudio ya que, dos años después de la entrada de HNA en el capital de NH, ambas empresas establecieron una joint venture para operar hoteles en China.

Nuestro estudio también presenta algunas limitaciones. En primer lugar, la inherente al propio proceso de obtención de información, ya que no se consiguió el mismo nivel de profundidad en las respuestas de todos los entrevistados. Cabe destacar la dificultad para conseguir datos primarios mediante entrevistas a altos directivos de empresas chinas derivada, no solo de su tradicional carácter reservado, sino también del hecho de que, en el momento de iniciar los contactos, sus operaciones en España eran muy recientes y todavía estaban en proceso de consolidación. Este hecho precisamente nos lleva a una segunda limitación del trabajo: no haber abordado en profundidad las dificultades post-adquisición provocadas por las diferencias culturales o por eventuales desavenencias entre empresa adquirente y adquirida. De hecho, con posterioridad a la realización de las entrevistas, se produjo una enorme disputa entre HNA y NH debido a que HNA, siendo ya accionista principal de $\mathrm{NH}$, adquirió la cadena Carlson-Rezidor, rival directo de $\mathrm{NH}$ en varios destinos europeos. Este conflicto de intereses derivó en la expulsión de los representantes de HNA en el consejo de administración de NH. Tras varios meses de disputas jurídicas, lo cierto es que HNA decidió poner a la venta su participación en $\mathrm{NH}$.

Estas limitaciones abren algunas vías para la investigación futura. Así, resultaría interesante analizar las mencionadas dificultades post-adquisición en el proceso de integración de las empresas hoteleras españolas dentro de los grupos inversores chinos. En este contexto, destaca especialmente el impacto de las diferencias culturales sobre los objetivos estratégicos de las empresas implicadas o sobre la gestión de los recursos humanos, en particular, la adaptación del personal español al estilo de gestión de las empresas chinas. También sería importante estudiar los efectos inducidos de la entrada de capital chino en las empresas hoteleras españolas, en términos de incremento del número de turistas chinos hacia España o de las oportunidades de negocio en China para la parte española.

Finalmente, sería necesario investigar el impacto de la nueva normativa del gobierno chino para regular las IDEs efectuadas por empresas chinas (State Council, 2017). Esa nueva normativa pretende racionalizar las adquisiciones internacionales, para evitar un excesivo endeudamiento de las empresas chinas que pueda afectar negativamente a la estabilidad financiera de China. Con ello, se han establecido tres categorías: inversiones 
incentivadas (aquellas que están en línea con los objetivos estratégicos de China), inversiones restringidas (aquellas que, debido a su elevado riesgo, podrían poner en peligro dichos objetivos) e inversiones prohibidas (aquellas que van en contra de los intereses nacionales). Cabe destacar que los sectores de turismo, ocio y actividades inmobiliarias han quedado encuadrados dentro de la categoría de inversiones restringidas, por lo que van a precisar un proceso de verificación previo por parte de las autoridades chinas. Una de las empresas que se ha podido ver afectada por esta nueva normativa es precisamente HNA, que está poniendo a la venta su participación en algunas empresas extranjeras, como la cadena hotelera norteamericana Hilton o la propia $\mathrm{NH}$, objeto de nuestro estudio.

\section{BIBLIOGRAFÍA}

ANAND, N., GARDNER, H.K. y MORRIS, T. (2007): "Knowledge-based innovation: Emergence and embedding of new practice areas in management consulting firms", Academy of Management Journal, vol. 50, $\mathrm{n}^{\circ} 2$, pp. 406-428.

ANDREU, R., CLAVER, E. y QUER, D. (2009): “China: ¿oportunidad o amenaza para el turismo español?”, Boletín Económico de Información Comercial Española, n ${ }^{\circ} 2958$, pp. 25-35.

BUCKLEY, P,J., CLEGG, L.J., CROSS, A.R., LIU, X., VOSS, H. y ZHENG, P. (2007): "The determinants of Chinese foreign direct investment", Journal of International Business Studies, vol. 38, n 4, pp. 499-518.

BUCKLEY, PJ., YU, P., LIU, Q., MUNJAL, S. y TAO, P. (2016): “The institutional influence on the location strategies of multinational enterprises from emerging economies: Evidence from China's cross-border mergers and acquisitions", Management and Organization Review, vol. 12, $\mathrm{n}^{\circ} 3$, pp 425-448.

CARRIL, F. y MILGRAM, J. (2016): "From Beijing to Madrid: Profiles of Chinese investors in Spain", Universia Business Review, n ${ }^{\circ}$ 51, pp. 112-129.

CASABURI, I. (2016): Tendencias de la Inversión China en Europa. Informe 2016-2017. ESADE China Europe Club. Barcelona.

CHILD, J. y MARINOVA, S. (2014): "The role of contextual combinations in the globalization of Chinese firms", Management and Organization Review, vol. 10, $\mathrm{n}^{\mathrm{o}} 3$, pp. 347-371.

CHILD, J. y RODRIGUES, S. B. (2005): "The internationalization of Chinese firms: A case for theoretical extension?", Management and Organization Review, vol. 1, n 3 , pp. 381-410.

CUERVO-CAZURRA, A. (2012): "Extending theory by analyzing developing country multinational companies: Solving the Goldilocks debate", Global Strategy Journal, vol. 2, no 3, pp. 153-167.

DIKOVA, D. y VAN WITTELOOSTUIJN, A. (2007): "Foreign direct investment mode choice: Entry and establishment modes in transition economies", Journal of International Business Studies, vol. 38, n 6, pp. 1013-1033.

DUANMU, J.L. (2014): "State-owned MNCs and host country expropriation risk: The role of home state soft power and economic gunboat diplomacy", Journal of International Business Studies, vol. 45, no 8, pp. 1044-1060. 
DUNNING, J.H. (1980). “Toward and eclectic theory of international production: Some empirical tests", Journal of International Business Studies, vol. 11, n 1, pp. 9-31.

DUNNING, J.H. (1988): "The eclectic paradigm of international production: A restatement and some possible extensions", Journal of International Business Studies, vol. $19, \mathrm{n}^{\mathrm{o}} 1$, pp. 1-32.

DUNNING, J.H. (1995): "Reappraising the eclectic paradigm in the age of alliance capitalism", Journal of International Business Studies, vol. 26, n 3, pp. 461-493.

DUNNING, J.H. (2000): "The eclectic paradigm as an envelope for economic and business theories of MNE activity", International Business Review, vol. 9, n 1, pp. 163-190.

DUNNING, J.H. (2006): “Comment on Dragon multinationals: New players in 21st century globalization”, Asia Pacific Journal of Management, vol. 23, nº 2, pp. 139-141.

DUNNING, J.H. y McQUEEN, M. (1982a): "Multinational corporations in the international hotel industry», Annals of Tourism Research, vol. 9, n ${ }^{\circ}$, pp. 69-90.

DUNNING, J.H. y McQUEEN, M. (1982b): "The eclectic theory of the multinational enterprise and the international hotel industry", en RUGMAN, A.M. (Ed.): New Theories of the Multinational Enterprise. Croom Helm. London, pp. 79-106.

EISENHARDT, K.M. (1989): "Building theories from case-study research", Academy of Management Review, vol. 14, n $\mathrm{n}^{\mathrm{4}}$, pp. 532-550.

EISENHARDT, K.M. y GRAEBNER, M.E. (2007): “Theory building from cases: Opportunities and challenges", Academy of Management Journal, vol. 50, no 1, pp. 25-32.

GE, G.L. y DING, D.Z. (2008): “A strategic analysis of surging Chinese manufacturers: The case of Galanz", Asia Pacific Journal of Management, vol. 25, n 4, pp. 667-683.

GE, G.L. y DING, D.Z. (2009): "The effects of the institutional environment on the internationalization of Chinese firms". En ALON, I. et al. (Eds.), China Rules. Globalization and Political Transformation. Palgrave MacMillan. Hampshire, pp. 46-68.

GLOBERMAN, S. y SHAPIRO, D. (2009): "Economic and strategic considerations surrounding Chinese FDI in the United States", Asia Pacific Journal of Management, vol. 26, no 1 , pp. 163-183.

HENNART, J.F. (2012): "Emerging market multinationals and the theory of the multinational enterprise", Global Strategy Journal, vol. 2, no 3, pp. 168-187.

HERNÁNDEZ, A. y ÁLVAREZ, O. (2009): “China, nuevo actor de la inversión extranjera global”, Boletín Económico de Información Comercial Española, no 2972, pp. 165-175.

HERNÁNDEZ, E. (2018): "El turismo chino en España: oportunidad y nuevas tendencias", Real Instituto Elcano, ARI 48/2018, pp. 1-8.

INE (2018): Estadística de Movimientos Turísticos en Frontera-Frontur. Instituto Nacional de Estadística. Madrid [www.ine.es].

JOHANSON, J. y VAHLNE, J.E. (1977): "The internationalization process of the firm. A model of knowledge development and increasing foreign market commitments", Journal of International Business Studies, vol. 8, n 1, pp. 23-32.

JOHANSON, J. y VAHLNE, J.E. (1990): “The mechanism of internationalization", International Marketing Review, vol. 7, $\mathrm{n}^{\circ}$ 4, pp. 11-24.

JOHANSON, J. y VAHLNE, J.E. (2009): “The Uppsala internationalization process model revisited: From liability of foreignness to liability of outsidership", Journal of International Business Studies, vol. 40, nº 9, pp. 1411-1431. 
JOHANSON, J. y WIEDERSHEIM-PAUL, F. (1975): "The internationalization of the firm. Four Swedish cases", Journal of Management Studies, vol. 12, no 3, pp. 305-322.

KEDIA, B., GAFFNEY, N. y CLAMPIT, J. (2012): "EMNEs and knowledge-seeking FDI", Management International Review, vol. 52, n ${ }^{\circ}$ 2, pp. 155-173.

KIM, J.U., y AGUILERA, R.V. (2016): "Foreign location choice: Review and extensions", International Journal of Management Reviews, vol. 18, n² 2, pp. 133-159.

KOLSTAD, I. y WIIG, A. (2012): "What determines Chinese outward FDI?", Journal of World Business, vol. 47, $\mathrm{n}^{\circ} 1$, pp. 26-34.

LI, P.P. (2007): "Toward an integrated theory of multinational evolution: The evidence of Chinese multinational enterprises as latecomers". Journal of International Management, vol. $13, \mathrm{n}^{\circ} 3$, pp. 296-318.

LIU, X., XIAO, W. y HUANG, X. (2008): "Bounded entrepreneurship and internationalisation of indigenous Chinese private-owned firms", International Business Review, vol. $17, \mathrm{n}^{\circ} 4$, pp. 488-508.

LOJO, A. (2016): "Chinese tourism in Spain: An analysis of the tourism product, attractions and itineraries offered by Chinese travel agencies", Cuadernos de Turismo, $\mathrm{n}^{\circ}$ 37, pp. 243-268.

LOJO, A. y LI, M.M. (2018): "Segmentation by experiential familiarity and travel mode of the Chinese outbound market to Spain", Journal of China Tourism Research, vol. $14, \mathrm{n}^{\circ} 1$, pp. 100-121.

LUO, Y. y TUNG, R.L. (2007): "International expansion of emerging-market enterprises: A springboard perspective", Journal of International Business Studies, vol. 38, n 4 , pp 481-498.

LUO, Y. y TUNG, R.L. (2018): “A general theory of springboard MNEs”, Journal of International Business Studies, vol. 49, $\mathrm{n}^{\circ}$ 2, pp. 129-152.

MATHEWS, J.A. (2002): "Competitive advantages of the latecomer firm: A resourcebased account of industrial catch-up strategies", Asia Pacific Journal of Management, vol. 19, nº 4, pp. 467-488.

MATHEWS, J.A. (2006): "Dragon multinationals: New players in 21st century globalization", Asia Pacific Journal of Management, vol. 23, n ${ }^{\circ}$ 1, pp. 5-27.

NARULA, R. (2012): "Do we need different frameworks to explain infant MNES from developing countries?”, Global Strategy Journal, vol. 2, n 3, pp. 188-204.

QUER, D., CLAVER, E. y RIENDA, L. (2015): “Chinese outward foreign direct investment: A review of empirical research", Frontiers of Business Research in China, vol. 9, no 3 , pp. 326-370.

QUER, D., CLAVER, E. y RIENDA, L. (2017): “Chinese multinationals in Spain: Determinants of establishment mode choice", Cuadernos de Gestión, vol. 17, $\mathrm{n}^{\circ} 2$, pp. 15-36.

RAMASAMY, B., YEUNG, M. y LAFORET, S. (2012): "China's outward foreign direct investment: Location choice and firm ownership", Journal of World Business, vol. 47, $\mathrm{n}^{\mathrm{o}} 1, \mathrm{pp} .17-25$.

RUGMAN, A.M. (2010): "Book review: Globalization of Chinese enterprises", The International Trade Journal, vol. 24, nº 3, pp. 352-354. 
SÁEZ, L., (2010): “Chinese outward direct investments in Spain”. International Economics Programme Paper 2010/06. Chatam House. London.

SANTACANA, R. y WANG, Y.J., (2008): "Privatización e internacionalización: las empresas chinas en España”, IX Congreso Internacional de la Asociación Española de Historia Económica. Murcia. Septiembre.

STATE COUNCIL (2017): Guidelines on Further Guiding and Regulating the Directions of Outbound Investments. State Council of the People's Republic of China. Beijing [www.gov.cn].

STRANGE, R. (2018): "Corporate ownership and the theory of the multinational enterprise”. International Business Review. vol. 27, n 6, pp. 1229-1237.

UNCTAD (2018): World Investment Report 2018. Investment and New Industrial Policies. United Nations Conference on Trade and Development. New York and Geneva.

WALSH, J.P., WANG, E. y XIN, K.R. (1999): "Same bed, different dreams: Working relationships in Sino-American joint ventures", Journal of World Business, vol. 34, $\mathrm{n}^{\circ} 1$, pp. 69-93.

YIN, R.K. (1981): "The case study crisis: Some answers", Administrative Science Quarterly, vol. 26, $\mathrm{n}^{\mathrm{o}} 1$, pp. 58-65.

YIN, R.K. (2014): Case Study Research: Design and Methods. $5^{\text {th }}$ edition. Sage. Thousand Oaks, CA. 
